5. - Applications of functional cohomology operations to the calculus of $\pi_{2 n+i}(U(n))$ for $i=6$ and 7, $n \geqq 4$, Mem. Fac. Sci. Kyushu Univ. Ser. A 17 (1963), 29-62.

6. R. E. Mosher, Some stable homotopy of complex projective space, Topology (to appear).

7. M. Rothenberg, The J functor and the nonstable homotopy groups of the unitary groups, Proc. Amer. Math. Soc. 15 (1964), 264-271.

8. H. Todo, $A$ topological proof of the theorems of Bott and Borel-Hirzebruch for homotopy groups of unitary groups, Mem. Coll. Sci. Kyoto 32 (1959), 109-119.

9. H. Toda, Composition methods in homotopy groups of spheres, Ann. of Math. Studies 49, Princeton Univ. Press, Princeton, N. J., 1962.

California State College at long Beach and

NORTHWESTERN UNIVERSITY

\title{
SOME PROPERTIES OF DISTRIBUTIONS WHOSE PARTIAL DERIVATIVES ARE REPRESENTABLE BY INTEGRATION ${ }^{1}$
}

BY HERBERT FEDERER

Communicated August 21, 1967

We denote $n$ dimensional Euclidean space by $R^{n}$ and let $H^{m}$ be $m$ dimensional Hausdorff measure.

It is well known that distributions of the type described in the title may alternately be characterized as corresponding to $H^{n}$ measurable real valued functions $f$ with the following property: There exists a sequence of infinitely differentiable real valued functions $f_{j}$ on $R^{n}$ such that

$$
\lim _{j \rightarrow \infty} \int_{K}\left|f_{j}-f\right| d H^{n}=0 \text { and } \liminf _{j \rightarrow \infty} \int_{K}\left\|D f_{j}\right\| d H^{n}<\infty
$$

for every compact subset $K$ of $R^{n}$. The class of such functions $f$ is now widely regarded as the proper generalization to $n>1$ of the class of those functions on $R$ which are $H^{1}$ equivalent to functions with finite total variation on every compact interval. However up to now there has been lacking an extension to $n>1$ of the basic classical results describing the continuity properties of functions with locally finite variation, namely that the set of points of discontinuity is countable and that one-sided limits exist everywhere. At first sight such an

1 This work was supported in part by a research grant from the National Science Foundation. 
extension to $n>1$ seems impossible, because $f$ may be $H^{n}$ essentially unbounded, and every function $H^{n}$ equivalent to $f$ may be discontinuous everywhere. The following new results show that the problem can be solved through use of $H^{n}$ approximate upper and lower limits, and the $n-1$ dimensional measure $H^{n-1}$.

For each $x \in R^{n}$ we let $\lambda(x)$ and $\mu(x)$ be the lower and upper $H^{n} a p$ proximate limits of $f$ at $x$; we recall that $\lambda(x)$ equals the least upper bound (possibly $\infty$ or $-\infty$ ) of the set of all real numbers $t$ such that

$$
\lim _{r \rightarrow 0+} r^{-n} H^{n}\left[R^{n} \cap\{z:|z-x| \leqq r, f(z) \leqq t\}\right]=0 .
$$

Defining

$$
\begin{aligned}
A & =\{x:|\lambda(x)|=\infty \text { or }|\mu(x)|=\infty\}, \\
B & =\{x:-\infty<\lambda(x)<\mu(x)<\infty\}, \\
g(x) & =[\lambda(x)+\mu(x)] / 2 \text { for } x \in R^{n} \sim A,
\end{aligned}
$$

and asuuming $n>1$ we prove:

(1) $H^{n-1}(A)=0$.

(2) $H^{n-1}$ almost all of $B$ can be covered by a countable family of $n-1$ dimensional regular, proper submanifolds of class 1 of $R^{n}$.

(3) For $H^{n-1}$ almost all $x$ in $R^{n} \sim B$,

$$
\lim _{r \rightarrow 0+} r^{-n} \int_{\{z:|z-x| \leqq r\}}|f(z)-g(x)|^{n /(n-1)} d H^{n} z=0 .
$$

(4) For $H^{n-1}$ almost all $x$ in $B$ there exists a real valued linear function $\alpha$ on $R^{n}$ such that

$$
\lim _{r \rightarrow 0+} r^{n} \int_{P(r)}|f(z)-\lambda(x)|^{n /(n-1)} d H^{n} z=0
$$

with $P(r)=\{z:|z-x| \leqq r$ and $\alpha(z-x) \geqq 0\}$, and

$$
\lim _{r \rightarrow 0+} r^{-n} \int_{N(r)}|f(z)-\mu(x)|^{n /(n-1)} d H^{n} z=0
$$

with $N(r)=\{z:|z-x| \leqq r$ and $\alpha(z-x) \leqq 0\}$.

(5) For $H^{n-1}$ almost all $x$ in $R^{n}$,

$$
g(x)=\lim _{r \rightarrow 0+} \int_{R^{n}} f(x) r^{n} k\left(r^{-1}|z-x|\right) d H^{n} z
$$

whenever $k$ is a real valued $H^{1}$ measurable function on $R$ with compact support such that 


$$
\int_{R^{n}} k(|z|) d H^{n} z=1 \text { and } \int_{R^{n}}|k(|z|)|{ }^{n} d H^{n} z<\infty
$$

The proposition (5) exhibits $g$ as a very attractive representative of the $H^{n}$ equivalence class of $f$, because arbitrary rotationally symmetric regularizations of $f$ converge to $g$ not only $H^{n}$ almost everywhere, but $H^{n-1}$ almost everywhere! (The corresponding proposition for $n=1$ asserts convergence $H^{0}$ almost everywhere, which means everywhere, and is classical; in this case $g$ has locally finite total variation and $\lambda(x), \mu(x)$ equal the one-sided limits of $g$ at $x$.) There remains the problem of generalizing (5) to certain functions $k$ with noncompact support, in particular to determine the smallest exponent needed to reproduce $g$ from its Fourier transform $H^{n-1}$ almost everywhere by spherical Riesz-Bochner summation (see [1]), provided $f$ behaves suitably at infinity. The rotationally symmetric approach is necessary because the directions of the half-spaces occurring in (4) depend on $x$.

Proofs of the above propositions will appear in the author's forthcoming book "Geometric measure theory". Our arguments make use of the set

$$
H=\left(R^{n} \times R\right) \cap\{(x, y): y \leqq \mu(x)\},
$$

whose essential boundary (for the purpose of the Gauss-Green theorem) is $H^{n}$ almost equal to the set

$$
C=\left(R^{n} \times R\right) \cap\{(x, y): \lambda(x) \leqq y \leqq \mu(x)\},
$$

which can $H^{n}$ almost be covered by a countable family of $n$ dimensional regular, proper submanifolds of class 1 of $R^{n} \times R$. From the geometric properties of these sets we obtain analytic properties of $f$ by studying the $n$ dimensional normal current $T$ in $R^{n}$ (see [3]) defined by the formula

$$
T(\phi)=\int_{R^{n}} f \wedge \phi
$$

for every infinitely differentiable differential form $\phi$ of degree $n$ on $R^{n}$ with compact support. For example, we show that

$$
\|\partial T\|(W)=\int_{R} H^{n-1}[W \cap\{x: \lambda(x) \leqq y \leqq \mu(x)\}] d H^{1} y
$$

for every Borel subset $W$ of $R^{n}$. (For the special case when $f$ is linearly continuous in the sense of [4], this formula has been obtained independently by a different method in [5].) Our approach also yields a 
new proof of the following result of [2]: For $H^{n}$ almost all $x$ in $R^{n}$ there exists a real valued linear function $\beta$ on $R^{n}$ such that

$$
\lim _{r \rightarrow 0+} r^{-n} \int_{\{z:|z-x| \leq r\}} \epsilon(z)^{n /(n-1)} d H^{n} z=0
$$

with $\epsilon(z)=|f(z)-f(x)-\beta(z-x)| /|z-x|$.

The author wishes to thank Casper Goffman for some stimulating discussions about the subject of this note, in particular for raising questions concerning the nature of the sets $A, B$ and $C$. Regarding the class of functions studied in [4] one can now assert the equivalence of the four properties: (I) $f$ is essentially linearly continuous. (II) $g$ is linearly continuous. (III) $H^{n-1}(B)=0$. (IV) $\|\partial T\|(W)=0$ whenever $W \subset R^{n}$ with $H^{n-1}(W)<\infty$.

\section{REFERENCES}

1. S. Bochner, Summation of multiple Fourier series by spherical means, Trans Amer. Math. Soc. 40 (1936), 175-207.

2. A. P. Calderon and A. Zygmund, On the differentiability of functions which are of bounded variation in Tonelli's sense, Rev. Un. Mat. Argentina 20 (1960), 102-121.

3. H. Federer and W. H. Fleming, Normal and integral currents, Ann. of Math. (2) 72 (1960), 458-520.

4. C. Goffman, $A$ characterization of linearly continuous functions whose partial derivatives are measures, Acta Math. 117 (1967), 165-190.

5. W. Ziemer, The area and variation of linearly continuous functions, Proc. Amer. Math. Soc. (to appear).

BROWN UNIVERSTTY 